OPEN ACCESS

Citation: R. Biagioli, J. Gonzalez-Monteagudo, C. Silva (2021) Editoriale. II ruolo delle madri nella prevenzione della radicalizzazione dei giovani. Prospettive internazionali di ricerca educativa. Rief 18, 2: pp. 5-16. doi: https://doi. org/10.36253/rief-12357.

Copyright: (c) 2021 E. Pelizzari. This is an open access, peer-reviewed article published by Firenze University Press (https:// oaj.fupress.net/index.php/rief) and distributed under the terms of the Creative Commons Attribution License, which permits unrestricted use, distribution, and reproduction in any medium, provided the original author and source are credited.

Data Availability Statement: All relevant data are within the paper and its Supporting Information files.

Competing Interests: The Author(s) declare(s) no conflict of interest.

\section{Il ruolo delle madri nella prevenzione della radicalizzazione dei giovani. Prospettive internazionali di ricerca educativa}

\author{
Raffaella Biagioli ${ }^{1}$, José Gonzalez-Monteagudo ${ }^{2}$, Clara Silva ${ }^{3,4}$
} 1. Radicalizzazione, educazione, genere: la funzione del sostegno
alla genitorialità

Con la Call all'origine del dossier di questo numero, la «Rivista Italiana di Educazione Familiare» ha voluto richiamare l'attenzione della Pedagogia sul fenomeno della radicalizzazione da un punto di vista educativo; fenomeno che ha prodotto in anni recenti gravi atti di violenza terroristica in varie parti del mondo, con un elevato numero di vittime.

In Europa, come in altri Continenti, si è assistito impotenti a un'escalation di attentati connessi al radicalismo islamico e le istituzioni, comprese quelle educative, hanno iniziato a domandarsi quali siano le cause di atti così violenti contro gli altri, ma spesso anche contro sé stessi. Il radicalismo islamico è stato così interpretato come una risposta al fallimento socioeconomico e alla marginalizzazione culturale (Ferrero, 2005). E sebbene non si debba far coincidere radicalismo con terrorismo, in quanto molti radicalizzati non mettono in atto azioni violente e terroristiche,

${ }^{1}$ Professoressa associata di Pedagogia generale e sociale presso il Dipartimento di Formazione, Lingue, Intercultura, Letterature e Psicologia (FORLILPSI) dell'Università degli Studi di Firenze.

${ }^{2}$ Ricercatore presso la Facoltà di Educazione dell'Università di Siviglia, coinvolto nei Progetti europei e invitato in atenei europei e americani, ha pubblicato su teorie educative contemporanee, diversità culturale, migrazioni, mediazione interculturale, ricerca qualitativa, etnografia.

${ }^{3}$ Professoressa ordinaria di Pedagogia generale e sociale presso il Dipartimento di Formazione, Lingue, Intercultura, Letterature e Psicologia (FORLILPSI) dell'Università degli Studi di Firenze.

${ }^{4}$ Questo contributo è il risultato del lavoro coordinato e congiunto dei tre Autori. Tuttavia, sono da attribuire a Clara Silva il par. 1, a Raffaella Biagioli il par. 2, e a José Gonzalez-Monteagudo il par. 3 (d'ora in avanti, laddove non diversamente specificato, le note a piè di pagina sono da intendersi a cura degli Autori, N.d.R.). 
è evidente che è all'interno dei processi di radicalizzazione che matura l'impegno terroristico (Laurano, Anzera, 2017).

È peraltro emerso che all'origine della decisione di diventare dei terroristi prevalgono motivazioni emotive, sociali e di status rispetto a fattori intellettuali e religiosi (Bartlett, Miller, 2012). Motivazioni simili hanno spinto anche alcuni europei convertiti all'Islam ad abbracciare posizioni radicali e a partecipare ad azioni terroristiche (Karagiannis, 2012). Vi è accordo, tra gli studiosi, nell'individuare nello sradicamento sociale e culturale dei migranti e soprattutto dei loro figli, nella loro esposizione a pratiche di discriminazione e di emarginazione sociale, nei problemi di identità che ne derivano (si veda la teoria della "doppia assenza» in Sayad, 1999, trad. it. 2002, passim), i fattori che contribuiscono alla fascinazione che i messaggi estremisti esercitano sulle giovani generazioni con background culturale e familiare migratorio (Waldmann, Sirseloudi, Malthaner, 2010).

Di qui una serie di proposte per contrastare la radicalizzazione nei giovani al fine di prevenire il terrorismo, anche per evitare misure estreme come quella di impedire il rientro in patria di coloro che sono andati in Medio Oriente a seguire corsi di addestramento per farsi reclutare nelle organizzazioni terroristiche (Lombardi et al., 2015). Ancora recentemente, però, gli studiosi lamentano il prevalere di una prospettiva securitaria nelle pratiche di contrasto alla radicalizzazione, mentre azioni efficaci necessitano di un approccio multidisciplinare, in cui tener conto degli strumenti messi a disposizione anche dalla Psicologia sociale (Meringolo, ed., 2020).

A livello internazionale, nondimeno, sempre più si insiste sulla necessità di combattere l'estremismo violento attraverso le istituzioni educative formali, rafforzando al contempo la resilienza della comunità con azioni di sostegno alle famiglie e ai gruppi più vulnerabili (Veenkamp, Zeiger, 2015). L'educazione è stata pertanto intesa come un vero e proprio «strumento di controterrorismo" ${ }^{5}$ (Webber, 2011, passim). Fino a pochi anni fa, tuttavia, la ricerca sui fenomeni di radicalizzazione da un punto di vista pedagogico era ancora molto limitata (Pels, de Ruyter, 2012), sebbene sia da tempo chiaro che l'azione di prevenzione rispetto ai processi di radicalizzazione deve essere svolta sia nell'ambito dell'educazione informale sia in quello formale, nell'intento di rafforzare l'identità delle giovani generazioni, favorendo l'inclusione sociale e culturale loro e delle loro famiglie. Negli ultimi anni, in questa prospettiva, sono stati messi a punto modelli di intervento rivolti a minori o giovani adulti radicalizzati, talora in stato di detenzione, fondati sull'approccio narrativo, sul medium teatrale e su giochi di ruolo (Gavrilovici, Dronic, 2020; Syrbe, 2020).

Benché le ricerche sulla radicalizzazione abbiano messo in luce che non esiste un solo profilo di radicalizzato, è significativo notare che gli attentati commessi in Europa sono stati compiuti da giovani di età media intorno ai 27 anni e che la loro morte è altamente contemplata nella loro azione criminale (Ibidem). Più nello specifico, due profili sono prevalenti nella letteratura scientifica sul fenomeno: a) il giovane "disintegrato" (secondo il paradigma illustrato in Djouder, 2007, trad. it. 2007) e "desocializzato" che vive nella marginalità sociale ed è soggetto a fenomeni di discriminazione; b) il giovane dotato di un solido curriculum formativo ma che non si sente rappresentato dalla società in cui vive e ne rifiuta i valori di riferimento contrapponendovi quelli dell'Islam radicale (Zanetti, 2018, p. 175). Questo significa che siamo di fronte a giovani che di fronte al complesso processo di costruzione della propria identità non hanno trovato supporto adeguato né in famiglia, né a scuola, né in altri contesti sociali. Di qui l'interiorizzazione di una immagine di sé così negativa da essere disposti ad annullarsi pur di sentirsi parte di qualcosa

\footnotetext{
${ }^{5}$ La traduzione italiana dell'espressione in oggetto è da intendersi a cura degli Autori, N.d.R.
} 
(l'Islam idealizzato, che non ha niente a che fare con quello dei genitori): la radicalizzazione, quindi, è da loro intesa come un'opzione estrema.

Le seconde, le terze generazioni sono così esposte a una costruzione di sé connessa a un senso di instabilità e frustrazione. Ciò tende a svuotare di significato anche il loro percorso scolastico, indebolendo quella spinta progettuale che nell'età adolescenziale è un fattore fondamentale per uno sviluppo equilibrato. Ne consegue il loro avvertirsi come una sorta di "corpo estraneo" della società, con relativa svalutazione del contributo che possono dare alla crescita culturale, economica e sociale.

La debolezza dei legami sociali e la mancanza di un senso di appartenenza solido portano dunque alcuni adolescenti ad allontanarsi dalla scuola, ad assumere atteggiamenti di ribellione assai pericolosi, che li spingono verso la devianza e la marginalizzazione sociale. Un esempio sotto gli occhi di tutti è quello dei giovani di background islamico che aderiscono alle promesse del messaggio jihadista globale (Vidino, Marone, Entenmann, 2017).

Tra i vari contesti educativi, l'ambiente familiare è quello che, prima degli altri, è chiamato a svolgere un lavoro preventivo nello sviluppo della radicalizzazione, indirizzando i figli fin da piccoli ad assumere atteggiamenti costruttivi di apertura prosociale e di dialogo verso l'altro. Occorre allora che i genitori siano aiutati a sviluppare adeguate competenze sul fenomeno della radicalizzazione affinché possano assumere un ruolo attivo nella promozione di attitudini positive verso la non-violenza. Da questa angolatura le madri, in particolare, possono assumere un ruolo chiave nella prevenzione degli estremismi violenti e nella messa in pratica di azioni educative mirate. Madri che necessitano di uno specifico aiuto e sostegno, se si considera che il fenomeno di radicalizzazione si associa spesso a un tessuto familiare meno saldo, dove la figura paterna può essere assente, e dunque in famiglie monoparentali dove è presente solo la madre (Khosrokhavar, 2017).

$\mathrm{La}$ «RIEF», proponendo la Call su Women's Role in Preventing Radicalization and Antisocial Behaviour in Family Contexts, ha sollecitato il mondo dell'educazione a interrogarsi sui contesti che generano il fenomeno della radicalizzazione e a riflettere su percorsi di ricerca che considerino la dimensione di genere come categoria fondamentale per analizzare un fenomeno così complesso, oggi attivo anche e soprattutto sul web e nei social media (Guazzini, Stefanelli, Imbimbo, 2020). Il fenomeno della radicalizzazione interseca questioni di carattere religioso, sociale, economico e culturale, e nel momento in cui viene collegato con la dimensione della differenza di genere ci spinge a soffermarci su aspetti spesso trascurati come il ruolo delle donne nella lotta contro il terrorismo o, al contrario, quello da loro svolto nelle organizzazioni terroristiche, dove la loro presenza sta aumentando, al di là dei numeri piuttosto contenuti relativi alle donne attentatrici (Vidino, Marone, Entenmann, 2017).

È dunque di fondamentale importanza il sostengo alla genitorialità, per far sì che le madri assumano il proprio ruolo genitoriale con maggior sicurezza, prevenendo le vulnerabilità e facendo, soprattutto, leva sulle loro potenzialità. Lo stesso Progetto "CommUnity - Build Communities, Create Peace" (https://www.thecommunityproject.eu) ${ }^{6}$, attraverso le varie attività che ha realizzato (Women's Community, Men's Community, Harmony Community), i cui risultati sono illustrati nel dossier di questo fascicolo, ha cercato di far dialogare tra loro donne, uomini e giovani, tentando di abbattere le barriere derivanti dalle differenze culturali, religiose e generazionali.

Gli obiettivi che il Progetto si è posto sono infatti quelli di rafforzare il senso di coesione sociale per prevenire e contrastare la radicalizzazione e gli estremismi violenti tra i giovani,

\footnotetext{
${ }^{6}$ Data di ultima consultazione: 10.11.21, N.d.A.
} 
supportare gli attori locali e le agenzie di formazione nella prevenzione della radicalizzazione tra i gruppi sociali maggiormente vulnerabili, promuovere il dialogo interculturale e interreligioso attraverso forme diverse di espressioni artistiche e culturali. L'isolamento e il mancato riconoscimento sociale comportano una crescente vulnerabilità delle donne alle lusinghe della propaganda jihadista che le espone ai rischi della radicalizzazione. Importante anche pensare alle madri che educano i loro bambini tra le sbarre e favorire quelle azioni progettuali che si interroghino e che riflettano sui contesti educativi dei bambini che sono portatori di diritti e che potrebbero crescere come marginali. La potenzialità insita nelle madri di compiere una trasmissione culturale rassicurante e, allo stesso tempo, aperta al dialogo e di educare alla costruzione e negoziazione di identità multiple va protetta e stimolata attraverso iniziative di sostegno alla genitorialità rivolte alle madri stesse.

All'interno di tali iniziative, la trasmissione culturale va incoraggiata, partendo dal riconoscimento della validità delle modalità e delle strategie di trasmissione messe in atto dalle madri. Una volta riconosciute, sarà possibile stimolarle attraverso la creazione di spazi al di fuori dell'ambito domestico dove non solo sia possibile mettere in atto le proprie pratiche culturali, ma anche stimolare una riflessione profonda e un confronto sui significati delle pratiche stesse. Si rende quanto mai urgente superare i limiti del multiculturalismo, per innestare processi di condivisione e di co-costruzione dei significati e dei sistemi simbolici di riferimento: in altre parole, la negoziazione adattiva è il presupposto necessario per prevenire irrigidimenti e ibernazioni identitarie.

Ugualmente, occorre valorizzare l'elemento dinamico, di trasformazione, che caratterizza ogni cultura, e storicizzarne la fenomenologia: la cultura non è un universo statico ma un mondo in trasformazione ed evoluzione, nel dialogo costante con l'altro. Da questa prospettiva, la radicalizzazione è uno dei principali antagonisti dei processi interculturali in quanto nel proporre una visione statica, uniforme e polarizzata del mondo, offre una soluzione identitaria ancorata a un rigido senso di appartenenza religioso e sociale.

Sicuramente il Progetto "CommUnity" ha contribuito a una maggiore consapevolezza dell'importanza della famiglia nei processi educativi di prevenzione della radicalizzazione e degli estremismi e maggiore consapevolezza del ruolo delle donne sia nei processi di arruolamento sia come soggetti che facilitano la prevenzione a partire dall'osservazione dei processi di micro-radicalizzazione che quotidianamente viviamo a casa, al lavoro, nei processi formativi che si creano nella scuola.

\section{Ruolo materno e cittadinanza}

Prevenire la radicalizzazione è senza dubbio un problema insidioso e complesso. Ci sono molti punti di vista contrastanti sulla radicalizzazione e sulle sue motivazioni. I giovani che si radicalizzano appartengono sia a ceti sociali poveri, emarginati, sia a contesti agiati. Comprendere e prevenire i fattori di spinta e di attrazione tra i giovani è una questione complessa che richiede l'intervento e la sinergia di molteplici agenzie formative, così come lo è comprendere il ruolo strategico della famiglia nell'educare i figli ad atteggiamenti costruttivi di apertura prosociale e di dialogo verso l'altro, per prevenire comportamenti antisociali e violenti.

Parlare di estremismo e radicalizzazione fa pensare già a giovani adulti o quasi, al disagio adolescenziale e giovanile, e a come intervenire in questa fascia di età, trascurando l'infanzia, mentre molti contributi che vanno a comporre questo fascicolo hanno offerto significativi riferimenti al ruolo dell'educazione familiare, sin dai primi anni di vita, nella prevenzione degli estremismi e dei comportamenti violenti. Da parte della ricerca pedagogica, fino ad oggi, risulta 
scarsa attenzione al ruolo fondamentale delle madri, delle figure femminili e alla differenza di genere nei processi di radicalizzazione e di arruolamento.

Il conflitto fra ruolo materno e diritti di cittadinanza, cioè tra la donna-madre e la donna-cittadina è stato molto forte in passato nella storia italiana, che non si allontana molto da quella di molti Stati europei, ma, forse, non è del tutto superato neppure oggi, perlomeno a livello simbolico. Forse questo potrebbe spiegare l'origine di comportamenti sociali individuali che riguardano le donne in cui la marginalità femminile, non più basata sul pregiudizio di genere, continua a persistere, attraverso processi secolari inalterabili di strutture organizzative funzionali al principio dell'immodificabilità del ruolo sessuale (Bolognari, 1997).

Simonetta Ulivieri ci ricorda che le donne sono le agenti primarie della socializzazione infantile, i soggetti principali della trasmissione dei ruoli sociali e dei modelli culturali. Questa attività risulta tuttavia, in molti casi, magari in forma irrazionale, legata alla tradizione e alle sue certezze e sicurezze, cosicché il discorso educativo più che a trasmettere con opportune correzioni e necessarie autocorrezioni il costume educativo, tende al contrario a riprodurre il passato (Ulivieri, 2007). Il ruolo sociale che, di nuovo, viene convenzionalmente assegnato alla donna è quello di proteggere i familiari e vigilare su di essi, "tenere le redini" della prole, mantenere un contatto con i fratelli e le sorelle, con la madre, con la suocera. Il materno può essere inteso così come un margine che, sebbene mitizzato, è spazio e condizione di confinamento, entro cui le donne continuano a essere ricondotte, indipendentemente dalla loro appartenenza geografica, culturale, religiosa e sociale.

La possibilità che le donne-madri sperimentino una dimensione di pratiche collettive in grado di riconoscerle e disposte ad ascoltarle e sostenerle come soggetti "pieni" - non solo madri (ed eventualmente straniere, per provenienza culturale e/o religiosa) ma cittadine: con diritti e non solo doveri; capaci di apprendere ma anche di insegnare - si connette intimamente alle finalità di prevenzione alla radicalizzazione e contribuisce in modo significativo e coerente alla declinazione delle pratiche educative correlate.

L'analisi in ottica di genere, applicata agli studi in materia di terrorismo e deradicalizzazione, ha dato negli ultimi anni un apporto significativo alla ricerca pedagogica, come hanno evidenziato alcuni contributi che considerano le donne e in particolare le madri una leva efficace delle progettualità a carattere preventivo.

La pedagogia della famiglia ha il compito di osservare ed elaborare le pratiche del quotidiano familiare per orientarle verso un percorso di senso e di ragione. Da una parte l'educazione familiare si pone come il luogo dell'analisi delle tecniche e delle pratiche per l'esercizio di una genitorialità consapevole e generante il senso dell'essere uomo, dall'altra, la pedagogia della famiglia ha il più alto compitò di indicare il percorso di senso della formazione di un soggetto. La pedagogia del familiare [...] tenta una rielaborazione dei contenuti e dei modelli che devono indirizzare i singoli membri della famiglia a "far-si famiglia", formandosi alla soggettività, trasformandosi come persone, divenendo uomini dotati di piena consapevolezza alla vita e all'essere, prima ancora che all'esistere e a 1 sopravvivere. Con l'ottica pedagogica assume un senso particolarmente simbolico la formazione dell'uomo che nasce nel rapporto madre-bambino, che nasce nella famiglia (Boffo, 2012, p. 98).

Da alcuni anni alle famiglie e alle madri è infatti riconosciuto un ruolo centrale per la precoce individuazione e la gestione di processi di radicalizzazione dei propri figli e delle proprie figlie. Si tratta di un riconoscimento che sembra intervenire in modo positivo sulla possibilità di ampliare quel ristretto e stereotipato margine definitorio entro cui, nel discorso pubblico il profilo delle donne (giovani o adulte, madri e non) consegue ancora a essere ricompreso, convocandolo su un piano sociale, civile e politico. E un riconoscimento che sembra infatti consentire l'apertura verso un immaginario più ampio che, con riguardo alle donne migranti (o 
di seconda e terza generazione) e quelle musulmane in particolare, sembra costituire un invito alla possibilità di pensar loro non solo come a oggetti di un discorso (politico, sociale, pedagogico) ma come soggetti cui riconoscere un ruolo positivo, rompendo i margini di un canone che tende frequentemente a costruirle e rappresentarle, alternativamente, come vittime che necessitano di essere salvate (poiché donne, oppresse da un punto di vista culturale e religioso e sfruttate dal terrorismo) o carnefici (in ragione della loro fede).

Esiste una responsabilità storica delle donne che come madri, come educatrici, come insegnanti, in passato hanno nettamente fatto prevalere un genere a danno di un altro, il loro. Nella pratica di ogni giorno, nell'insegnare il silenzio, la rassegnazione, o nel favorire e sottolineare in positivo alcune attività, quelle svolte dai bambini e dai ragazzi, la donna si fa responsabile verso le proprie simili di una precisa oppressione culturale, trasmettendo veti, abitudini mentali alla dipendenza, alla subalternità che difficilmente la bambina divenuta donna riuscirà a superare, e che andranno ad alimentare, in una spirale perversa, nuove future oppressioni e soggezioni. Questo processo non è certo casuale, né legato a fattori "naturali", secondo cui le donne sarebbero portatrici biologicamente inferiori di contenuti culturali (Ulivieri, 2007, pp. 169-170).

Come i femminismi hanno da tempo messo in luce, una certa costruzione e valorizzazione del materno ha prodotto una solida e radicata essenzializzazione del femminile, che si è trovata a coincidere con le sole dimensioni di riproduzione biologica e di cura. È questo un vincolo che si rintraccia e rinnova nelle pratiche di vita quotidiana, dentro e fuori gli interni familiari e che, non a caso, interessa le stesse politiche sociali, dove l'assenza di un riconoscimento economico a compensazione delle attività di cura connesse alla riproduzione del quotidiano le sottintende obblighi a carico delle famiglie e, in particolare, delle donne, sebbene evidentemente essenziali alla sopravvivenza e al funzionamento dei sistemi familiari, economici e sociali. Un obbligo che resta tuttavia implicito perché, appunto, culturalmente ricondotto entro la dimensione del naturale, facendo apparire "normale" e "fisiologica" la funzione di cura femminile.

Sotto questo punto di vista occorre fare attenzione a non cadere nello stereotipo che ritiene le donne maggiormente inclini e adatte per la cura in ambito familiare, professionale e sociale, altrimenti finiamo per far ricadere tale incombenza esclusivamente sul femminile. Donne e madri rischiano infatti di essere racchiuse in una definizione che vede loro imputato il ruolo, dirimente, di sole e uniche custodi dei destini delle proprie figlie e figli; e, a seconda degli esiti, etero- e auto-valutate come "brave o cattive" madri, "innocenti o colpevoli".

L'esperienza di cura genitoriale vissuta dalle donne, oggetto dell'interesse degli interventi di prevenzione alla radicalizzazione, ha modo di trovare un riconoscimento e una valorizzazione che vadano oltre il considerarla punto di partenza e luogo entro cui, di necessità, inserire formazioni e informazioni volte a rendere le donne edotte e in grado di riconoscere i segni di un possibile accostamento dei propri figli e figlie al terrorismo. Un punto di partenza che rischia, infatti, di inscrivere il proprio obiettivo educativo e formativo entro un'immagine di sola insufficienza e di problematicità. Si tratta, viceversa, della possibilità di conoscere e ri-conoscere le pratiche di cura e di genitorialità concretamente vissute all'interno delle specificità di contesti territoriali, dove le singole biografie (individuali e familiari) si trovano immerse, relazionandosi, obbligatoriamente con alterne fortune, alle complessità presenti; permettendo la valorizzazione delle loro diverse espressioni; accogliendone i chiaroscuri, le problematicità, le fatiche (Biagioli, 2015).

È forse in questo modo che il "margine" può diventare luogo di resistenza, all'interno di progettualità educative che non si limitino a convocare le donne destinatarie degli interventi, e a farlo in ragione della loro funzione materna, nel tentativo di renderle "sentinelle" competenti ed efficienti nell'ordine della tutela dei propri figli e figlie. Nel considerarle prioritariamente 
cittadine-donne, queste progettazioni possono costruire e presidiare tempi e luoghi entro cui le esperienze, le voci e le narrazioni delle donne che coinvolgono, possono apportare un significativo e dirimente contributo alla costruzione di osservatori critici sul presente e di spazi di supporto a una loro attivazione civile e politica, procedendo ben oltre le finalità preventive previste.

Prevenzione primaria: famiglia, come amplificatore dei messaggi e delle conoscenze. Essa può rappresentare una risorsa poiché favorisce la rielaborazione dell'identità originaria in una nuova forma, più rispondente alle esigenze di individui che si muovono tra orizzonti culturali diversi.

Prevenzione secondaria, dove la famiglia diventa, in prima linea, il "campanello di allarme" per intercettare in anticipo e leggere gli indizi che possono allertarci sui pericoli di adesione a prospettive estremiste e violente, da parte di giovani o adulti. La famiglia può assolvere il ruolo di partner nelle azioni di educazione, di gestione dei processi informali di apprendimento che possono generare resilienza e prospettive di pensiero più aperte, permeabili e inclusive.

Prevenzione terziaria, perché la famiglia può essere già il setting dentro il quale progettare l'intervento; o, ancora, come testimoniato da alcune esperienze internazionali, può diventare uno dei "poli di comunità di pratiche", al cui interno tentare di recuperare persone che abbiano compiuto atti violenti. È questo il livello più complesso dove si intrecciano, con maggiore chiarezza, processi di security e di rieducazione, tra pratiche di intervento formali e informali.

All'interno di queste premesse, le progettualità ricordate possono costituire un invito e uno stimolo, ma anche una concreta opportunità, al sostegno delle madri, affinché prendano parola su di sé, sulla propria esperienza di genitrici, donne e cittadine, tornando a tenere insieme il privato e il politico, sui canoni correnti di genitorialità, occidentali e non, attesi e previsti, sulle loro origini, i loro limiti e le problematicità.

Una presa di parola che può costituire lo spazio per un confronto paritetico, dove donne dalle differenti origini e provenienze culturali e sociali possano incontrarsi e confrontarsi, non solo prendendo parte attiva e critica all'analisi pedagogica e di genere di modelli, ruoli e aspettative connesse alla genitorialità, al genere e alla cittadinanza (Silva, 2017), ma alla loro stessa decostruzione, trasformazione ed evoluzione.

Si tratta di obiettivi ambiziosi, che richiedono il supporto di servizi e istituzioni in grado di combattere stereotipi androcentrici ed etnocentrici e affrontare il peso e la complessità derivanti dal considerare le donne non come soggetti utili o necessari per obiettivi di sicurezza nazionale e/o internazionale, e nei rispetti di questi supinamente aderenti; soggetti da formare $o$, al più, da consultare, ma soggettività il cui profilo è politico, attivo, plurale e, auspicabilmente, critico.

\section{Presentazione dei contributi}

Tra i contributi che costituiscono questo numero vi sono riflessioni di carattere teoretico sul ruolo delle donne, in particolare le madri, come figure-chiave nella prevenzione del disagio giovanile e dei comportamenti estremisti dei giovani; soprattutto, sono riportate indagini empiriche - insieme a esempi di buone pratiche - accompagnate da cornici di riferimento teoriche e supportate da un'esplicazione della metodologia di ricerca usata, sul tema della prevenzione della radicalizzazione tra i giovani e sull'importanza del ruolo preventivo e supportivo delle famiglie e delle madri.

Elisa Pelizzari, nell'articolo La donna in Africa e il suo ruolo educativo in seno alla famiglia: un possibile baluardo contro la radicalizzazione dei piu giovani? Il caso della regione sahelo-sahariana, affronta la questione del radicalismo giovanile sulle base di alcune ricerche che Lei stessa conduce in Mali, Senegal e Repubblica di Guinea. In questi Paesi l'estremismo assume una connotazione prevalentemente religiosa e riguarda il fondamentalismo islamico che arruola 
molti giovani in nome di un'adesione totalizzante a un messaggio considerato portatore della più alta verità divina.

$\mathrm{Al}$ di là del contesto geografico, l'attenzione del contributo si concentra sulla questione educativa, poiché attraverso la trasmissione di valori e modelli comportamentali si forgiano le menti dei giovani e si influenzano le loro scelte di vita, che potranno rappresentare un punto di continuità o di rottura con le tradizioni familiari. In queste famiglie la figura materna apparentemente poco visibile nella società è in realtà molto più importante e influente di quanto non possa sembrare a una prima analisi.

In From Radicals to Community Builders: Reframing the Roles of Women through Design Thinking, Patricia Huion, Handan Sayer e Raquel Pinto Pinto-Bello Gomez problematizzano il concetto di radicalizzazione. In particolare, affrontano la questione della radicalizzazione femminile attraverso la metodologia del design thinking, che cerca di proporre possibili soluzioni progettuali a problemi complessi. L'articolo è accompagnato da una riflessione critica che evidenzia la necessità urgente di agire sui ruoli femminili radicalizzati e sconosciuti, poiché questi aspetti oscuri facilitano la realizzazione di comportamenti radicali. La prospettiva di genere appare indispensabile nelle politiche e negli approcci per prevenire la radicalizzazione. Entrambi i generi soffrono di punti di vista distorti nella definizione del problema della prevenzione della radicalizzazione, poiché ignorare la "mascolinità tossica" nelle strategie di prevenzione rende le misure di prevenzione inefficienti, così come considerare solo i ruoli femminili di donne come vittime passive e casalinghe. Il contributo cerca di trovare delle strategie per dare spazio alle storie di tutte le donne - passive, alleate, radicalizzate - legate a gruppi radicali di destra o di sinistra, a gruppi monotematici o politico-religiosi, e trasformando le loro problematiche in capacità di agire come agenti di prevenzione.

Hakan Ayçiçek e Patricia Huion, in Not All Migrant Men Embrace Toxic Masculinity, Do They?, mettono al centro dell'attenzione la figura ambigua dell'uomo migrante, che racchiude vari stereotipi. Vengono descritti vari modelli di identità maschili, con una particolare attenzione alla mascolinità tossica e alle scelte che gli uomini migranti devono affrontare nel processo di integrazione.

Un'ulteriore questione importante che il contributo sottolinea è il nesso tra violenza di genere e situazioni di violenza familiare vissute nell'infanzia. Gli uomini possono evitare la natura tossica della mascolinità solo se vengono educati dalla loro famiglia e dalla società all'idea che occorre essere rispettosi, enfatici, premurosi, accettanti e gentili con tutti gli esseri umani, e che questo sia un punto di forza molto più grande. Secondo gli Autori, quanto detto sopra dovrebbe essere insegnato ai ragazzi migranti e alle loro famiglie attraverso adeguati programmi di sostegno alla genitorialità.

Nel contributo dal titolo Né vittime, né carnefici. Cittadine. Un'analisi pedagogica dei ruoli delle madri nei percorsi di prevenzione alla radicalizzazione violenta e al terrorismo, Lisa Brambilla propone un'analisi pedagogica nell'ottica di genere sul ruolo delle madri nei percorsi di prevenzione ai processi di radicalizzazione dei giovani, offrendo significativi orientamenti per la strutturazione di interventi a carattere preventivo. L'articolo analizza i limiti e i rischi connessi al ruolo di prevenzione riconosciuto alle madri, indicando possibilità per esplorarne le potenzialità e cercando sempre di sottolineare la necessità e l'importanza di dare sostegno e pieno riconoscimento al percorso di cittadinanza attiva e critica delle donne nella società.

In Preventing Marginalization and Radicalization through Theatre and the Expressive Arts: Empowering Intercultural Dialogue, Francesco Pipparelli focalizza l'attenzione sul teatro e sull'arte come forme artistiche per promuovere il dialogo interculturale, facilitare il processo di coesione e inclusione sociale, e ridurre il rischio di comportamenti antisociali violenti fra i giovani. Il 
teatro e l'arte sono da sempre usati come forme e mezzi di narrazione, per generare emozioni e far immedesimare il pubblico nelle storie che sente, o alle quali assiste. Per questo appaiono particolarmente adatti come strumenti di inclusione e prevenzione della marginalizzazione. Il contributo fa riferimento ad alcune forme di laboratori teatrali e di racconto, specialmente quelli per donne migranti, che rappresentano ottimi casi di facilitazione del processo di scoperta e definizione della propria identità in modo costruttivo. Tali percorsi rappresentano il punto di partenza verso l'integrazione tramite l'arte, facilitando il processo di consapevolezza e inclusione dei, e in particolare delle, partecipanti.

Claudio Melacarne in Prospettive teoriche per prevenire i processi di micro-radicalizzazione. Il ruolo della famiglia e dei contesti parentali, presenta un saggio declinato in due parti. Nella prima viene delineato lo stato dell'arte del problema della radicalizzazione, con particolare riferimento agli episodi di radicalizzazione della vita quotidiana; nella seconda, l'attenzione viene invece, focalizzata sul ruolo preventivo della radicalizzazione e della radicalizzazione violenta, a livello primario, secondario e terziario. Per quanto concerne la prevenzione primaria, la famiglia diventa non solo un contesto di fruizione di saperi utili alla prevenzione, ma può configurarsi come amplificatore dei messaggi e delle conoscenze. A livello secondario, la famiglia rappresenta il primo "campanello di allarme" per intercettare preventivamente e leggere i segnali di allerta sui pericoli di adesione a prospettive estremiste e violente da parte di giovani o adulti. A livello della prevenzione terziaria, nei processi di "disingaggio", la famiglia può essere il contesto entro cui progettare l'intervento o, come testimoniato da alcune esperienze internazionali, può diventare uno dei principali "poli di comunità". L'articolo si conclude con un'analisi della letteratura nazionale e internazionale, oltre che con una disamina sui possibili livelli di intervento educativo in collaborazione con i contesti familiari.

Anja Baukloh fornisce una panoramica in Conflict capability e mediazione: un approccio preventivo alla radicalizzazione e ai comportamenti antisociali in contesti familiari e microsociali e una disamina su concreti interventi di analisi nelle dinamiche di conflitto microsociale, con particolare riferimento alla famiglia. Il punto di partenza è il concetto di conflict capability: ovvero, la capacità degli esseri umani di gestire in modo appropriato un conflitto. L'approccio presentato unisce uno sguardo sistemico al fenomeno e un accompagnamento trasformativo alle capacità delle singole persone, in particolare le donne, di affrontare i conflitti e cercare una soluzione. L'articolo propone anche una riflessione sull'importanza di una cultura diffusa della gestione costruttiva dei conflitti - la conflict capability, appunto - per rafforzare la pratica della democrazia nelle società contemporanee.

In Prevenire la radicalizzazione e i comportamenti antisociali. Madri e bambini nei non-luoghi dell'educabilità, Raffaella Biagioli affronta la questione della prevenzione della radicalizzazione a partire dalle madri nel contesto penitenziario. Nell'intersezione con altre dimensioni come quella etnica, religiosa e sociale, la differenza di genere spinge a soffermarsi su aspetti spesso trascurati e di far emergere il ruolo delle madri nei luoghi di reclusione che, assieme ai propri figli, rappresentano una popolazione a rischio per le difficoltà insite nella condizione di restrizione.

Per i bambini e le bambine, crescere in un contesto di reclusione e privazione come il carcere rappresenta sicuramente un fattore di rischio, che predispone al disagio giovanile e ai comportamenti violenti ed estremisti: secondo Michael Kimmel (2018), la chiave per la de-radicalizzazione è capire queste difficoltà e proporre delle possibilità di inserimento sociale, evitando marginalizzazioni che in futuro potrebbero indurre i bambini, una volta divenuti adolescenti e giovani, a ricercare l'inserimento radicalizzato all'interno di gruppi. Ancora una volta, la strategia più idonea risulta essere la prevenzione, per cercare di arginare tutti quei 
problemi che, inevitabilmente, riemergerebbero in un contesto di solitudine. Anche in questo caso, i minori possono essere aiutati agendo, prima di tutto, sulle loro famiglie.

Loretta Fabbri e Francesca Bracci presentano un articolo dal titolo Pratiche di interculturalismo quotidiano. Etnografia di un condomino multietnico, in cui indagano i processi di costruzione della differenza nella materialità di uno spazio di interculturalismo quotidiano attraverso l'analisi delle interazioni che scandiscono la vita di un condominio multietnico situato nel centro storico di Arezzo. Si tratta di uno studio etnografico che ha inglobato nel processo di analisi le esperienze e le interpretazioni personali delle Autrici, direttamente implicate e partecipi della realtà osservata in quanto residenti nel condominio. L'interesse è rivolto allo studio dell'uso pratico della differenza in contesti di interazione quotidiana, analizzando un condominio inteso come spazio effettivo di convivenza multietnica dove cose, persone, spazi, categorie e geografie si articolano lungo un continuum di senso ed esperienza, che ha trovato nel palazzo e nelle sue pratiche dell'abitare un laboratorio di apprendimento. La ricerca mette in evidenza quanto sia limitante e appartenga ad un livello di pensiero precritico ritenere che il nostro senso dell'ordine sia migliore di "altri"; questo chiama in causa l'importanza del relativismo culturale e l'esigenza di educare a un pensiero inclusivo, capace di muoversi entro un sistema di significato plurale, dove la diversità sia fonte di generazione di nuove pratiche funzionali a relazioni aperte a prospettive diverse, non necessariamente antitetiche.

Valentina Guerrini, in Donne immigrate, estremismi e radicalizzazione. Tra rischio di vulnerabilità e opportunità di divenire costruttrici di comunità, affronta la complessa questione dell'estremismo e della radicalizzazione dal punto di vista della differenza di genere evidenziando, nello stesso tempo, sia una partecipazione crescente delle donne nelle organizzazioni terroristiche negli ultimi anni, sia la forte presenza di forme di discriminazione a discapito delle donne all'interno delle organizzazioni stesse. Il contributo dedica un'attenzione particolare alle ragazze e alle donne musulmane, che come dimostrano varie ricerche, risultano essere particolarmente a rischio di discriminazione ed esclusione sociale. Sullo sfondo si afferma la necessità di attuare politiche e modelli educativi basati sull'inclusione, sul riconoscimento della differenza come valore, sull'accettazione dell'altro, sul dialogo e sull'empowerment femminili per sostenere le famiglie, e in particolare le madri, nella prevenzione del disagio giovanile e delle varie forme di estremismo e radicalizzazione.

Maria Grazia Proli, nel contributo Prevenire i comportamenti antisociali e la radicalizzazione dei giovani attraverso Internet. L'esperienza del Progetto europeo "DIVE IN", riflette sull'importanza della prevenzione dei comportamenti antisociali e della radicalizzazione nei giovani, soprattutto diffusi attraverso Internet e i social media. In questa prospettiva, e in riferimento ad alcuni documenti emanati dalle Istituzioni europee, si evidenzia la necessità di un rafforzamento delle competenze specifiche dei professionisti dell'educazione necessarie per saper rilevare i comportamenti potenzialmente pericolosi nei giovani e prevenire e contrastare la radicalizzazione. A tal proposito, l'articolo illustra gli obiettivi e i primi risultati del Progetto europeo "DIVE IN - Preventing violent radicalisation among young individuals in Europe by innovative training approaches", finalizzato alla prevenzione della radicalizzazione violenta nei giovani attraverso la formazione, in presenza e a distanza, di coloro che lavorano con questi ultimi.

Il numero, inoltre, accoglie una sezione miscellanea, formata da quattro contributi, che esulano dalle tematiche della Call per affrontare altre questioni attuali e ricche di interesse per la ricerca pedagogica oggi.

Giada Prisco in Transizione ecologica e nuove generazioni: il ruolo della famiglia nella promozione della sostenibilità in un'ottica inclusiva e interculturale, offre interessanti riflessioni sulle conseguenze provocate dall'emergenza sanitaria da Covid-19 sull'educazione, proponendo una 
valorizzazione del bene comune attraverso una transizione ecologica per realizzare comunità più sostenibili, inclusive, ecologiche e resilienti. La chiave di volta sembra essere proprio l'educazione, a partire dalla famiglia quale agenzia formativa per eccellenza, che oggi più che mai è chiamata in causa, in prima linea, per educare le giovani generazioni aduna coscienza planetaria, affinché possano affrontare in maniera democratica e resiliente le sfide presenti e future.

Flávio Santiago e Ana Lúcia Goulart de Faria, in Theatrical Scrawls. The Aesthetic Creations of Young Girls and Young Boys Scribble the Scene, focalizzano l'attenzione sulle attività teatrali nella prima infanzia come mezzi per creare relazioni paritarie tra bambini, bambine, e adulti. Viene presentato anche il lavoro di formazione svolto dalla Compagnia teatrale "La Baracca", insieme a educatrici e educatori dei nidi di Bologna. Gli approcci utilizzati, di tipo creativo, ludico e fantastico, sono molto efficaci nel catturare l'attenzione e favorire il coinvolgimento dei più piccoli, ma anche facilitare nuovi "modi di essere" degli educatori stessi.

Sara Mori, Silvia Panzavolta, Alessia Rosa in Distance Education and Parental Role, in Italy. Evidence-based Reflections from an International Survey, after the First Lockdown presentano i risultati di un'indagine internazionale sui modi in cui genitori e caregiver sono stati in grado di costruire la capacità di impegnarsi nell'apprendimento dei bambini durante il periodo di allontanamento sociale derivante dalla pandemia globale di Covid-19. Il sondaggio, coordinato dall'Università di Bath, ha coinvolto 23 Paesi partner, tra cui IUL che rappresenta l'Italia.

La pandemia ha posto diversi ostacoli che le famiglie hanno dovuto affrontare quotidianamente nel tentativo di educare i propri figli, e questo ha rappresentato un'opportunità storica unica per i ricercatori e i responsabili politici per comprendere tutte le lezioni impartite da questa emergenza globale e lavorare a stretto contatto con le famiglie, sostenendole nel processo di apprendimento dei bambini. Occorre quindi una co-progettazione educativa tra la famiglia e altre agenzie educative, promuovendo l'interconnessone di occasioni di apprendimento formale, informale e non-formale.

Infine, Alice Moscaritolo, Anne Dupuy, Pierre Ratinaud, Chantal Zaouche Gaudron, in Vécu de familles monoparentales avec jeunes enfants durant le premier confinement lié à la COVID-19 en France, affrontano la questione delle conseguenze derivanti dalle misure imposte durante la pandemia per le famiglie monoparentali, basandosi sull'analisi delle risposte di un campione di queste famiglie a un sondaggio online.

I dati confermano che le famiglie monoparentali si trovano in una situazione di maggiore fragilità rispetto alle altre coinvolte nel sondaggio: per i genitori soli, soprattutto le madri, il maggiore carico emotivo, educativo e di cura si è confrontato con la pressione percepita sulla cura dei loro figli, così come con percezioni meno soddisfacenti della propria genitorialità. Ciononostante, la maggior parte delle relazioni tra genitori e figli si è rafforzata, sebbene particolarmente sorprendente sia la mancata menzione della "rete sociale".

\section{Riferimenti bibliografici}

Badran M. (2013): Feminism in Islam: Secular and Religious Convergences. London: Oneworld.

Bartlett J., Miller C. (2012): The Edge of Violence: Towards Telling the Difference between Violent and Non-Violent Radicalization. Terrorism and Political Violence, 24(1), pp. 1-21.

Biagioli R. (2015): I significati pedagogici della scrittura e del racconto di sé. Napoli: Liguori.

Boffo V. (2012): Nella famiglia. In A. Anichini, V. Boffo, F. Cambi, A. Mariani, L. Toschi (a cura di): Comunicazione formativa. Milano: Apogeo.

Bolognari V. (1997): Educare alle pari opportunità. Esperienze e ricerche condotte a Messina. In S. Ulivieri (a cura di): L'educazione e i marginali. Scandicci (Fi): La Nuova Italia, pp. 235-251.

Djouder A. (2007): Disintegrati. Trad. it. Milano: il Saggiatore, 2007. 
Ferrero M. (2005): Radicalization as a Reaction to Failure: An Economic Model of Islamic Extremism. Public Choice, 122, pp. 199-220.

Gavrilovici O., Dronic A. (2020): Increasing Professionals' Knowledge and Skills to Deal with Violent Radicalisation. In P. Meringolo (ed.): Preventing Violent Radicalisation in Europe. Multidisciplinary Perspectives. Chaim: Springer, pp. 109-134.

Guazzini A., Stefanelli F., Imbimbo E. (2020): Further Examples of Violent Attitudes: The Online Radicalisation. In P. Meringolo (ed.): Preventing Violent Radicalisation in Europe. Multidisciplinary Perspectives. Chaim: Springer, pp. 191-208.

Iori V. (2008): Nuove fragilità e lavoro di cura. Milano: Unicopli.

Karagiannis E. (2012): European Converts to Islam: Mechanisms of Radicalization. Politics, Religion \& Ideology, 13(1), pp. 99-113.

Kimmel M. (2018): Healing from Hate: How Young Men Get Into-and Out of-Violent Extremism. Oakland (CA): University of California Press.

Khosrokhavar F. (2014): Radicalisation. Paris: Editions de la Maison des Sciences de l'Homme.

Laurano P., Anzera G. (2017): L'analisi sociologica del nuovo terrorismo tra dinamiche di radicalizzazione e programmi di de-radicalizzazione. Quaderni di Sociologia, 75, pp. 99-115.

Lombardi M., Ragab E., Chin V., Dandurand Y., de Vitiis V., Burato A. (ed.) (2015): Countering Radicalisation and Violent Extremism among Youth to Prevent Terrorism. Amsterdam: IOS Press.

Meringolo P. (ed.) (2020): Preventing Violent Radicalisation in Europe. Multidisciplinary Perspectives. Chaim: Springer.

Mussi A. (2019): Islamofobia al femminile tra pregiudizi e occasioni di dialogo. In L. Brambilla, A. Galimberti, S. Tramma (a cura di): Educazione e terrorismo. Milano: FrancoAngeli.

Pels T., de Ruyter D.J. (2012): The Influence of Education and Socialization on Radicalization: An Exploration of Theoretical Presumptions and Empirical Research. Child Youth Care Forum, 41, pp. 311-325.

Pepicelli R. (2010): Femminismo islamico. Corano, diritti, riforme. Roma: Carocci.

Sayad A. (1999): La doppia assenza. Dalle illusioni dell'emigrato alle sofferenze dell'immigrato. Trad. it. Milano: Raffaello Cortina, 2002.

Silva C. (2017): Cittadinanza. In M. Fiorucci, F. Pinto Minerva, A. Portera (a cura di): Gli alfabeti dell'intercultura. Pisa: ETS.

Syrbe H. (2020): Theatre Projects in Prisons. In P. Meringolo (ed.): Preventing Violent Radicalisation in Europe. Multidisciplinary Perspectives. Chaim: Springer, pp. 85-108.

Ulivieri S. (2007): Silences and Words of Women. Historia de la Educaciòn, n. 26, pp. 169-180.

Veenkamp I., Zeiger S. (2015): Countering Violent Extremism: Program and Policy Approaches Relating to Youth through Education, Families and Communities. In M. Lombardi, E. Ragab, V. Chin, Y. Dandurand, V. de Vitiis, A. Burato (eds.): Countering Radicalisation and Violent Extremism Among Youth to Prevent Terrorism. Amsterdam: IOS Press, pp. 151-163.

Vidino L., Marone F., Entenmann E. (2017): Jihadista della porta accanto. Radicalizzazione e attacchi jihadisti in Occidente. Milano: Ledizioni Ledi/Publ..

Waldmann P., Sirseloudi M., Malthaner S. (2010): Where Does the Radicalisation Process Lead? Radical Community, Radical Networks and Radical Subcultures. In M. Ranstorp (ed.): Understanding Violent Radicalisation. Terrorist and Jihadist Movements in Europe. London- New York: Routledge, pp. 50-67.

Webber D. (2011): Education as a Counterterrorism Tool and the Curious Case of the Texas School Book Resolution. University of Maryland Law Journal Race, Religion, Gender and Class, 11(2), pp. 271-292.

Zanetti M. (2018): Il fenomeno della radicalizzazione nei giovani: vulnerabilità ambientali, individuali e strategie di prevenzione. Formazione \& Insegnamento, a. XVI, n. 3, pp. 171-181. 